\title{
Radiative decays of light-quark mesons to a pion revisited in the covariant oscillator quark model
}

\author{
Kenji Yamada* \\ Department of Science and Manufacturing Technology, Junior College Funabashi Campus, \\ Nihon University \\ E-mail: vamada.kenjienihon-u.ac.jp

\section{Tomohito Maeda} \\ Department of Science and Manufacturing Technology, Junior College Funabashi Campus, \\ Nihon University \\ E-mail: maeda.tomohitodnihon-u.ac.ip
}

\begin{abstract}
The present talk consists of two parts. The first one is allocated for consideration of the dimension of bilocal meson fields for quark-antiquark meson systems, which have been so far treated as the bosonic fields independent of constituent quark flavors, in the covariant oscillator quark model. Revisiting the electromagnetic currents of quark-antiquark meson systems, we show that the bilocal meson fields should be bosonic for light-quark meson systems, while fermionic for heavy-light and heavy-heavy meson systems. In the second part we apply the effective electromagnetic interactions of meson systems derived in the first part to radiative decay processes for the excited states of light-quark mesons. The calculated results for the $\pi \gamma$ decay widths of the $a_{1}(1260), a_{2}(1320), b_{1}(1235)$ and $\pi_{2}(1670)$ mesons are in fair agreement with experiment, except for the $b_{1}(1235)$ meson. As for the $\rho(770)^{0} \gamma$ decay width of the $f_{1}(1285)$ the present model strongly supports the experimental results in 1995 and 2016, respectively, of VES and CLAS Collaborations, not the average value by the Particle Data Group.
\end{abstract}

XVII International Conference on Hadron Spectroscopy and Structure - Hadron2017

25-29 September, 2017

University of Salamanca, Salamanca, Spain

${ }^{*}$ Speaker. 


\section{Introduction}

In dealing with radiative decays some typical approximations, such as long wavelength and nonrelativistic approximations, which are not always justified, are usually used. For instance, the recoil effect of final-state mesons is neglected, though the momenta of those mesons are often comparable to their masses, especially in light-quark meson sectors. There is also ambiguity associated with a choice of the relativistic or nonrelativistic phase space in the nonrelativistic quark model. When the decays become relativistic, no one knows the rigorous way of deriving the relationship between nonrelativistic decay amplitudes and relativistic decay widths.

In the covariant oscillator quark model (COQM), on the other hand, hadrons themselves and their interactions are formulated in a manifestly covariant way. Since both the interaction operator and wave functions are relativistic, the gauge invariance is preserved and the conserved electromagnetic currents of hadrons are given explicitly in terms of the constituent quark variables [四].

\section{The covariant oscillator quark model}

Basic framework of the COQM. In the COQM quark-antiquark meson systems are described by the bilocal field

$$
\Psi\left(x_{1}, x_{2}\right)_{\alpha}{ }^{\beta}=\Psi(X, x)_{\alpha}{ }^{\beta},
$$

where $x_{1}^{\mu}\left(x_{2}^{\mu}\right)$ is the space-time coordinate, $\alpha(\beta)$ the Dirac spinor index of the constituent quark (antiquark), and the center-of-mass and relative coordinates are defined, respectively, by

$$
X^{\mu}=\frac{m_{1} x_{1}^{\mu}+m_{2} x_{2}^{\mu}}{m_{1}+m_{2}}, x^{\mu}=x_{1}^{\mu}-x_{2}^{\mu}
$$

with the constituent quark (antiquark) mass $m_{1}\left(m_{2}\right)$. The bilocal meson field is required to satisfy the Klein-Gordon-type equation

$$
\left(-\frac{\partial^{2}}{\partial X_{\mu} \partial X^{\mu}}-\mathscr{M}^{2}(x)\right) \Psi(X, x)_{\alpha}^{\beta}=0
$$

with the squared-mass operator, in the pure confining force limit,

$$
\mathscr{M}^{2}(x)=2\left(m_{1}+m_{2}\right)\left(\frac{1}{2 \mu} \frac{\partial^{2}}{\partial x_{\mu} \partial x^{\mu}}+U(x)\right), U(x)=-\frac{1}{2} K x_{\mu} x^{\mu}+\text { const. }
$$

where $\mu=m_{1} m_{2} /\left(m_{1}+m_{2}\right)$ is the reduced mass and $K$ is the spring constant. A solution of this equation can be written as

$$
\Psi(X, x)_{\alpha}^{\beta}=N e^{\mp i P_{\mu} X^{\mu}} \Phi(v, x)_{\alpha}^{\beta}, v^{\mu}=P^{\mu} / M
$$

where $N$ is the normalization constant for the plane wave, $P^{\mu}$ and $M$ are the center-of-mass momentum and mass, respectively, of the whole meson system, and $\Phi(v, x)_{\alpha}{ }^{\beta}$ is the internal wave function which is given by the direct product of eigenfunctions of the squared-mass operator and the Bargmann-Wigner spinor functions, defined by the direct tensor product of respective Dirac spinors, with the meson four velocity $v^{\mu}$, for the constituent quark and antiquark. 
Key features of the COQM. In order to freeze the redundant freedom of relative time for the four-dimensional harmonic oscillator, which gives here the squared-mass operator, the definitemetric-type subsidiary condition is adopted [వ]. The space-time wave functions satisfying this condition is normalizable and leads to the desirable asymptotic behavior of electromagnetic form factors of hadrons. The eigenvalues of the squared-mass operator are given by

$$
M_{N}^{2}=M_{0}^{2}+N \Omega, \Omega=2\left(m_{1}+m_{2}\right) \sqrt{\frac{K}{\mu}},
$$

where $N=2 N_{r}+L, N_{r}(L)$ being the radial (orbital angular momentum) quantum number. This squared-mass formula gives linear Regge trajectories with the slope $\Omega^{-1}$, in accord with the wellknown experimental fact.

\section{Electromagnetic currents for quark-antiquark meson systems}

The above Klein-Gordon-type equation is rewritten in terms of the quark and antiquark coordinates as

$$
2\left(m_{1}+m_{2}\right)\left(\sum_{i=1}^{2} \frac{-1}{2 m_{i}} \frac{\partial^{2}}{\partial x_{i \mu} \partial x_{i}^{\mu}}-U\left(x_{1}, x_{2}\right)\right) \Psi\left(x_{1}, x_{2}\right) \alpha^{\beta}=0 .
$$

This equation is derived from either of the actions

$$
S_{\text {free }}^{(\mathrm{KG}, \mathrm{S})}=\int d^{4} x_{1} \int d^{4} x_{2} \mathscr{L}_{\text {free }}^{(\mathrm{KG}, \mathrm{S})}\left(\Psi, \partial_{1 \mu} \Psi, \partial_{2 \mu} \Psi\right)
$$

with the Klein-Gordon-like Lagrangian

$$
\mathscr{L}_{\text {free }}^{(\mathrm{KG})}=\bar{\Psi}\left(x_{1}, x_{2}\right) 2\left(m_{1}+m_{2}\right)\left(\sum_{i=1}^{2} \frac{-1}{2 m_{i}} \frac{\overleftarrow{\partial}}{\partial x_{i \mu}} \frac{\vec{\partial}}{\partial x_{i}^{\mu}}-U\left(x_{1}, x_{2}\right)\right) \Psi\left(x_{1}, x_{2}\right)
$$

and the Schrödinger-like Lagrangian

$$
\mathscr{L}_{\text {free }}^{(\mathrm{S})}=\bar{\Psi}\left(x_{1}, x_{2}\right)\left(\sum_{i=1}^{2} \frac{-1}{2 m_{i}} \frac{\overleftarrow{\partial}}{\partial x_{i \mu}} \frac{\vec{\partial}}{\partial x_{i}^{\mu}}-U\left(x_{1}, x_{2}\right)\right) \Psi\left(x_{1}, x_{2}\right)
$$

where the bilocal field $\Psi\left(x_{1}, x_{2}\right)$ has the dimension of bosons $\left[\mathrm{M}^{1}\right]$ and fermions $\left[\mathrm{M}^{3 / 2}\right]$ for $\mathscr{L}_{\text {free }}^{(\mathrm{KG})}$ and $\mathscr{L}_{\text {free }}^{(\mathrm{S})}$, respectively, except for the dimension of internal wave functions $\left[\mathrm{M}^{2}\right]$.

The interaction of quark-antiquark meson systems with an electromagnetic field can be obtained [3] by the minimal substitutions

$$
\frac{\partial}{\partial x_{i}^{\mu}} \rightarrow \frac{\partial}{\partial x_{i}^{\mu}}+i e Q_{i} A_{\mu}\left(x_{i}\right)
$$

in the free Lagrangians $\mathscr{L}_{\text {free }}^{(\mathrm{KG}, \mathrm{S})}$, in which the heuristic prescription by Feynman, Kislinger and Ravndal [四] with some extension is adopted as the following replacements

$$
\frac{\overleftarrow{\partial}}{\partial x_{i \mu}} \frac{\vec{\partial}}{\partial x_{i}^{\mu}} \rightarrow\left(1-g_{M}^{(i)}\right) \frac{\overleftarrow{\partial}}{\partial x_{i \mu}} \frac{\vec{\partial}}{\partial x_{i}^{\mu}}+g_{M}^{(i)} \frac{\overleftarrow{\partial}}{\partial x_{i}^{\mu}} \gamma^{\mu} \gamma^{\nu} \frac{\vec{\partial}}{\partial x_{i}^{\nu}}
$$


where $Q_{i}(i=1,2)$ are the quark and antiquark charges in units of $e$ and $g_{M}^{(i)}$ are the parameters related to the anomalous magnetic moments of constituent quarks. Then the action for the electromagnetic interaction of meson systems is obtained, up to the first order of $e$, as

$$
S_{\mathrm{int}}^{(\mathrm{KG}, \mathrm{S})}=\int d^{4} x_{1} \int d^{4} x_{2} \sum_{i=1}^{2} j_{i}^{(\mathrm{KG}, \mathrm{S}) \mu}\left(x_{1}, x_{2}\right) A_{\mu}\left(x_{i}\right) \equiv \int d^{4} X J^{(\mathrm{KG}, \mathrm{S}) \mu}(X) A_{\mu}(X)
$$

with the conserved currents

$$
j_{i}^{(\mathrm{KG}) \mu}\left(x_{1}, x_{2}\right)=2\left(m_{1}+m_{2}\right)\left\langle\bar{\Psi}\left(x_{1}, x_{2}\right) \frac{-i e Q_{i}}{2 m_{i}}\left(\frac{\overleftrightarrow{\partial}}{\partial x_{i \mu}}-i g_{M}^{(i)} \sigma^{\mu \nu}\left(\frac{\vec{\partial}}{\partial x_{i}^{v}}+\frac{\overleftarrow{\partial}}{\partial x_{i}^{\nu}}\right)\right) \Psi\left(x_{1}, x_{2}\right)\right\rangle
$$

and

$$
j_{i}^{(\mathrm{S}) \mu}\left(x_{1}, x_{2}\right)=\frac{j_{i}^{(\mathrm{KG}) \mu}\left(x_{1}, x_{2}\right)}{2\left(m_{1}+m_{2}\right)}
$$

where $\langle\cdots\rangle$ means taking trace concerning the Dirac indices. The electric charges of meson systems are given by the diagonal elements

$$
Q_{\mathrm{meson}}^{(\mathrm{KG}, \mathrm{S})}=\int d^{3} X\left\langle i\left|J^{(\mathrm{KG}, \mathrm{S}) 0}(X)\right| i\right\rangle .
$$

The respective features of the Klein-Gordon-like and Schrödinger-like currents are as follows:

- The Klein-Gordon-like current. The meson charge is given by

$$
Q_{\mathrm{meson}}^{(\mathrm{KG})}=\left(Q_{1}+Q_{2}\right) e,
$$

which reproduces the physical one correctly. The currents $j_{i}^{(\mathrm{KG}) \mu}\left(x_{1}, x_{2}\right)$ have no absolute mass scales of constituent quarks. This would seem to be natural for light-quark systems from the viewpoint of QCD in the chiral limit.

- The Schrödinger-like current. In this case the meson charge becomes

$$
Q_{\text {meson }}^{(\mathrm{S})}=\frac{M}{m_{1}+m_{2}}\left(Q_{1}+Q_{2}\right) e,
$$

where $M$ is the meson mass. This expression does not generally coincide with the physical meson charge. However, it gives the correct charge in the heavy quark limit. This means that the Schrödinger-like current is applicable to heavy-light and heavy-heavy systems. If the meson masses for nonrelativistic quark systems are written as

$$
M_{n}=\left(m_{1}+m_{2}\right)+\mathscr{E}_{n}
$$

with the $n$-th excitation energy $\mathscr{E}_{n}$, then the meson charge can be expressed as

$$
Q_{\text {meson }}^{(\mathrm{S})}=\left(1+\frac{\mathscr{E}_{n}}{M_{0}}\right)\left(Q_{1}+Q_{2}\right) e
$$

where $M_{0}$ is a mass of the ground-state meson. From this expression it is found that the applicability of the schrödinger-like current is estimated by the ratio of the excitation energy to the ground-state mass. The currents $j_{i}^{(\mathrm{S}) \mu}\left(x_{1}, x_{2}\right)$, unlike $j_{i}^{(\mathrm{KG}) \mu}\left(x_{1}, x_{2}\right)$, have the absolute mass scales of constituent quarks, which is a desirable feature in describing the nonrelativistic quark systems. 
From the above considerations, it is concluded that the dimension of bilocal meson fields is bosonic for light-quark systems, while fermionic for heavy-light and heavy-heavy systems, except for the dimension of internal wave functions $\left[\mathrm{M}^{2}\right]$. Our recent studies of the pionic decays of excited heavy-light, charmed and charmed-strange, mesons support this conclusion for heavy-light systems [可].

\section{Radiative decay widths of light-quark mesons}

The above effective electromagnetic interactions $S_{\text {int }}^{(\mathrm{KG} S)}$ describe systematically all the electromagnetic interactions of quark-antiquark meson systems. For the radiative decays of light-quark mesons the decay width is obtained, following the usual procedure with the Klein-Gordon-like interaction $S_{\text {int }}^{(\mathrm{KG})}$, as

$$
\Gamma=\frac{1}{2 J_{i}+1} \frac{|\mathbf{q}|}{8 \pi M_{i}^{2}} \sum_{\text {spin }}\left|\mathscr{M}_{f i}\right|^{2}
$$

where $M_{i}\left(J_{i}\right)$ are the mass (spin) of the initial-state meson and $|\mathbf{q}|$ is the photon three-momentum. In the actual applications of the above formula to radiative decay widths of light-quark mesons the physical masses are used for initial- and final-state mesons, except for the pion.

Here we restrict ourselves to a discussion on the radiative decays of light-quark mesons only with nonstrange quarks. Then the present radiative decay model has two parameters, $\Omega$ and $g_{M}$ (三 $\left.g_{M}^{(u)}=g_{M}^{(d)}\right)$, the inverse of the Regge slope and the parameter related to the anomalous magnetic moment of $u$ and $d$ quarks. We take the value of $\Omega=1.14 \mathrm{GeV}^{2}$ and determine a value of $g_{M}$ so as to fit the experimental width for $\rho(770)^{ \pm} \rightarrow \pi^{ \pm} \gamma$. We also treat the pion mass as an additional parameter, which is determined in the following two ways:

- Case A. Assuming that the pion lies on the spin-singlet, $b_{1}(1235)-\pi_{2}(1670)$, trajectory, the effective pion mass becomes $0.476 \mathrm{GeV}$. Calculating the numerical width for $\rho(770)^{ \pm} \rightarrow$ $\pi^{ \pm} \gamma$ with this pion mass, $g_{M}=1.51$ is obtained.

- Case B. In the case of the pion and the $\pi_{2}(1670)$ lying on the same trajectory with $\Omega=$ $1.14 \mathrm{GeV}^{2}$, the effective pion mass is $0.719 \mathrm{GeV}$. In this case the width for $\rho(770)^{ \pm} \rightarrow \pi^{ \pm} \gamma$ is calculated with this pion mass only for the exponential factor of the formula, while the physical pion mass for the other part. This treatment gives $g_{M}=0.743$.

Numerical results of the radiative decay widths are shown in comparison with experiment in Table $\mathbb{W}$. The agreement with the measured widths for $\pi \gamma$ decays is satisfactory, though that for $\pi_{2}(1670)^{ \pm} \rightarrow \pi^{ \pm} \gamma$ less so in Case A, except for $b_{1}(1235)^{ \pm} \rightarrow \pi^{ \pm} \gamma$. It should be noted that only the electric (convection) current, independent of $g_{M}$, contributes to the decay processes $b_{1}(1235)^{ \pm} \rightarrow$ $\pi^{ \pm} \gamma$ and $\pi_{2}(1670)^{ \pm} \rightarrow \pi^{ \pm} \gamma$. In addition, the width for the decay $f_{1}(1285) \rightarrow \rho(770)^{0} \gamma$ can be calculated by just the same parameters, assuming that the $f_{1}(1285)$ is a pure $u \bar{u}+d \bar{d}$ state. The results are also shown in Table I, where the present model predictions are found to be in good agreement with the VES $[\square]$ and CLAS [ [ $]$ ] measurements, not the PDG average value. 
Table 1: Theoretical and experimental widths for radiative decays of light-quark mesons. The experimental values of the initial-state masses and radiative widths are taken from the Particle Data Group (PDG) [B], unless otherwise noted. As for the $\rho^{0} \gamma$ decay of the $f_{1}(1285)$, the VES-based value was obtained from the VES measurement of the branching fraction to $\rho^{0} \gamma[\square]$ and the PDG total width, while the CLAS-based one was computed using the CLAS measurement of the branching ratio $\Gamma\left(\rho^{0} \gamma\right) / \Gamma(\eta \pi \pi)$ []] and the PDG total width and branching fraction to $\eta \pi \pi$. The statistical and systematic errors of the respective measured values were added in quadrature.

\begin{tabular}{lcccc}
\hline \hline & & \multicolumn{2}{c}{ Theory (keV) } & \\
\cline { 3 - 4 } Decay process & $M_{i}(\mathrm{MeV})$ & Case A & Case B & Experiment (keV) \\
\hline$\rho(770)^{ \pm} \rightarrow \pi^{ \pm} \gamma$ & $775.26 \pm 0.25$ & 68 (fit) & 68 (fit) & $68 \pm 7$ \\
$a_{1}(1260)^{ \pm} \rightarrow \pi^{ \pm} \gamma$ & $1230 \pm 40$ & 464 & 394 & $640 \pm 246$ \\
$a_{2}(1320)^{ \pm} \rightarrow \pi^{ \pm} \gamma$ & $1318_{-0.6}^{+0.5}$ & 340 & 250 & $311 \pm 25$ \\
$b_{1}(1235)^{ \pm} \rightarrow \pi^{ \pm} \gamma$ & $1229.5 \pm 3.2$ & 42 & 52 & $230 \pm 60$ \\
$\pi_{2}(1670)^{ \pm} \rightarrow \pi^{ \pm} \gamma$ & $1672.2 \pm 3.0$ & 88 & 150 & $181 \pm 29$ \\
$f_{1}(1285) \rightarrow \rho(770)^{0} \gamma$ & $1281.9 \pm 0.5$ & 752 & 369 & $636 \pm 240$ [VES] \\
& & & & $545 \pm 253$ [CLAS] \\
& & & & $1203 \pm 331$ \\
\hline \hline
\end{tabular}

\section{Summary}

The dimension of bilocal meson fields should be bosonic for light-quark meson systems, while fermionic for heavy-light and heavy-heavy ones, except for the dimension of internal wave functions. The calculated results for the radiative decay widths of the $a_{1}(1260), a_{2}(1320), b_{1}(1235)$ and $\pi_{2}(1670)$ mesons to the pion are in fair agreement with experiment, aside from $b_{1}(1235)^{ \pm} \rightarrow \pi^{ \pm} \gamma$. For the decay $f_{1}(1285) \rightarrow \rho(770)^{0} \gamma$ the present model strongly supports the experimental results of VES and CLAS Collaborations.

\section{References}

[1] S. Ishida, K. Yamada and M. Oda, Phys. Rev. D 40, 1497 (1989).

[2] T. Takabayasi, Nuovo Cim. 33, 668 (1964).

[3] R. G. Lipes, Phys. Rev. D 5, 2849 (1972); S. Ishida and J. Otokozawa, Prog. Theor. Phys. 53, 217 (1975).

[4] R. P. Feynman, M. Kislinger and F. Ravndal, Phys. Rev. D 3, 2706 (1971).

[5] T. Maeda et al., AIP Conference Proceedings 1735, 050012 (2016); T. Maeda, K. Yamada and M. Oda, Pionic transitions of excited charmed mesons in the covariant oscillator quark model, in these proceedings.

[6] C. Patrignani et al. (Particle Data Group), Chin. Phys. C 40, 100001 (2016) and 2017 update.

[7] D.V. Amelin et al. (VES Collaboration), Z. Phys. C 66, 71 (1995).

[8] R. Dickson et al. (CLAS Collaboration), Phys. Rev. C 93, 065202 (2016). 